\title{
Human Endogenous Retrovirus-H Long Terminal Repeat-Associating Protein 2 Possesses Prognostic Significance and Promotes Progression of Papillary Thyroid Cancer
}

\author{
Yongzhi Niu', Yichuan Huang', Anbing Dong $\mathbb{D}^{2}$, Yinghe Sun ${ }^{2}$ \\ 'Department of Otolaryngology-Head and Neck Surgery, The Affiliated Hospital of Qingdao University, Qingdao, People's Republic of China; \\ ${ }^{2}$ Department of Thyroid Surgery, The Affiliated Hospital of Qingdao University, Qingdao, People's Republic of China
}

Correspondence: Yinghe Sun, Department of Thyroid Surgery, The Affiliated Hospital of Qingdao University, No. 16, Jiangsu Road, Qingdao, Shandong, People's Republic of China, Tel +86-532-82919570, Email syh_thyroid@I63.com

Purpose: Identification of novel biomarkers could benefit the clinical therapy and management of papillary thyroid carcinoma (PTC). Human endogenous retrovirus long terminal repeat-associating protein 2 (HHLA2) has been reported to play roles in the development of various cancers. The clinical significance and biological function of HHLA2 in PTC were investigated.

Patients and Methods: The expression level of HHLA2 was evaluated in PTC tissues (from 107 PTC patients) and cell lines (TPC1, IHH-4, CGTH-W3, and MDA-T32 cells) by RT-qPCR. The clinical significance of HHLA2 was estimated with a series of statistical analyses. The biological function of HHLA2 was assessed with the CCK8 assay and transwell assay.

Results: HHLA2 was upregulated in PTC compared with the normal samples and was associated with the positive lymph node metastasis and advanced TNM stage of PTC patients. HHLA2 was an independent prognostic factor associated with the poor survival of PTC patients. Additionally, HHLA2 functioned as a tumor promoter that enhanced the progression of PTC cells.

Conclusion: HHLA2 could serve as a prognostic biomarker and tumor promoter in PTC, providing a novel therapeutic target of PTC.

Keywords: papillary thyroid cancer, HHLA2, development, survival, prognosis

\section{Introduction}

With the continuously increasing incidence, thyroid cancer has gradually become the most common malignant neoplasm in the endocrine system. ${ }^{1}$ According to the pathological, clinical, and genetic features of patients, thyroid cancer pathologically contains papillary thyroid carcinoma (PTC), follicular thyroid carcinoma, anaplastic thyroid carcinoma, and medullary thyroid carcinoma. ${ }^{2-4}$ PTC is the most common histological type of thyroid cancer accounting for approximately $80 \% .{ }^{5}$ Recurrence and distant metastasis are major adverse events that threaten recovery and postoperative life quality of a proportion of PTC patients. ${ }^{6,7}$ Moreover, the traditional therapeutic means also limit the cure of PTC patients. It is critical to know the factors that indicate the course of cancer and the outcome of PTC patients.

With the development of molecular biology, numerous molecules have been identified as indicators for the tumor progression and disease development of various cancers. In PTC, a number of molecules were reported to serve as biomarkers that indicate the progression and predicate clinical outcomes. The certain novel B7 family is a member of these molecules, of which several members have been characterized to participate in the development of various cancers. For example, B7-H4 was associated with the tumor stage of ovarian serous carcinoma. ${ }^{8}$ B7-H5 was demonstrated to be involved in the mediating effect of EGFR in NSCLC. ${ }^{9}$ HHLA2 (B7-H7) is a recently identified member of the B7 family and has been revealed to suppress the function of human CD4 and CD8 T cells, which might be a novel therapeutic target 
of human cancers. ${ }^{10}$ In gastric cancer, the overexpression of HHLA2 was identified to represent the malignant status and the poor prognosis of patients. ${ }^{11}$ It was reported that HHLA2 was associated with the recurrence and survival of pancreatic and ampullary cancer patients. ${ }^{12}$ HHLA2 was also speculated to function as a biomarker of PTC to predict disease development and the patient's post-surgical prognosis. However, there were few data available that could demonstrate the function of HHLA2 in PTC.

The expression and function of HHLA2 in PTC was evaluated in this study with clinical tissues and cultured cells, aiming to appraise novel biomarker of PTC.

\section{Patients and Methods}

\section{Patients}

This study had obtained approval from the Ethics Committee of the Affiliated Hospital of Qingdao University and informed consent from every participant. All procedures followed were in accordance with the ethical standards of the responsible committee on human experimentation (institutional and national) and with the Helsinki Declaration of 1964 and later versions. A total of 107 PTC patients that underwent surgical resection in The Affiliated Hospital of Qingdao University were included in this study from January 2013 to December 2015. The tumor tissues and normal tissues (at least $2 \mathrm{~cm}$ from tumor tissues) were collected from enrolled patients and stored in liquid nitrogen. All patients were followed up for 5 years after their surgery to obtain their survival information.

\section{Real-Time Quantitative PCR (RT-qPCR)}

Total RNA was extracted from collected tissues and cultured cells by Trizol (Life Technologies, USA) and synthesized cDNA with a reverse transcription-polymerase chain reaction kit (TaKaRa, Japan) according to the manufacturers' instructions. The quantitation of HHLA2 (primer forward 5'-GGAACACTTCATTTTCCCCAATTC-3', reverse 5'TCTCCTACATGCTCTCCTTCCT-3') was performed with the help of the SYBR Green Master Mix kit and a GAPDH (primer forward 5'-CTGTCGGSCCTGGCCAAG-3', reverse 5'-CACCCTCGCCAAAGGTGA-3') as the internal reference. The $2^{-\triangle \Delta C T}$ method was used to calculate the expression level of HHLA2.

\section{Cell Culture and Transfection}

Four types of human PTC cell lines (TPC-1, IHH-4, CGTH-W3, and MDA-T32) and a normal thyroid cell line (HTORI3) were purchased from ATCC and cultured in the RPMI 1640 medium (Life Technologies, USA) with 10\% FBS (Life Technologies, USA). HHLA2 was silenced by the RNAi approach with the small hairpin RNA (shRNA) and was overexpressed by the transfection of lentivirus containing HHLA2 sequences. The transfection efficiency was evaluated by detecting the expression of HHLA2.

\section{Cell Proliferation Assay}

The proliferation of transfected cells was assessed by the CCK8 kit (Dojindo, Japan) according to the manufacturer's instructions. Cells were seeded into 96 -well plates at a density of $5 \times 10^{3}$ cells/well. The cells were supplied with the full culture medium and incubated at $37^{\circ} \mathrm{C}$ for $0,24,48$, and $72 \mathrm{~h}$. The CCK8 kit was added to each well at each time point. The absorbance at $450 \mathrm{~nm}$ was detected by a microplate reader to evaluate the cell proliferation of PTC.

\section{Cell Migration and Invasion Assay}

The 24-well transwell plates (Corning, USA) were applied in the migration and invasion assay. The cells were seeded into the upper chambers of the transwell at a density of $1 \times 10^{4}$ cells/well and supplemented with an FBS-free culture medium. The medium with $10 \%$ FBS was placed in the bottom chamber as a chemoattractant. The upper chamber was pre-coated with the Matrigel (Corning, USA) for the invasion evaluation. The plates were incubated at $37^{\circ} \mathrm{C}$ for $24 \mathrm{~h}$, then, the migrated cells and invaded cells in the bottom chamber were stained with $1 \%$ crystal violet and fixed with $4 \%$ paraformaldehyde. The cell number was counted with the Olympus inverted microscope. 
A

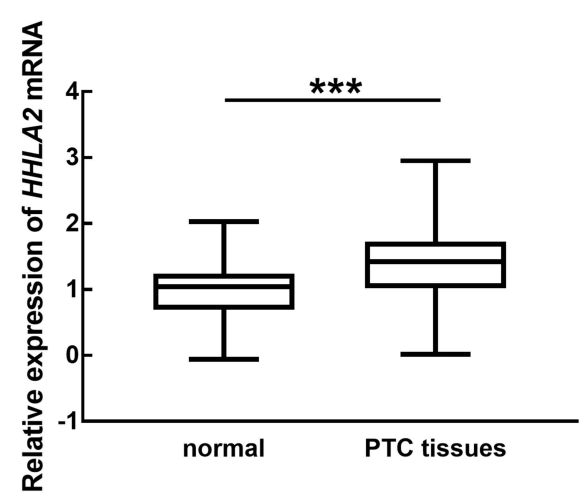

B

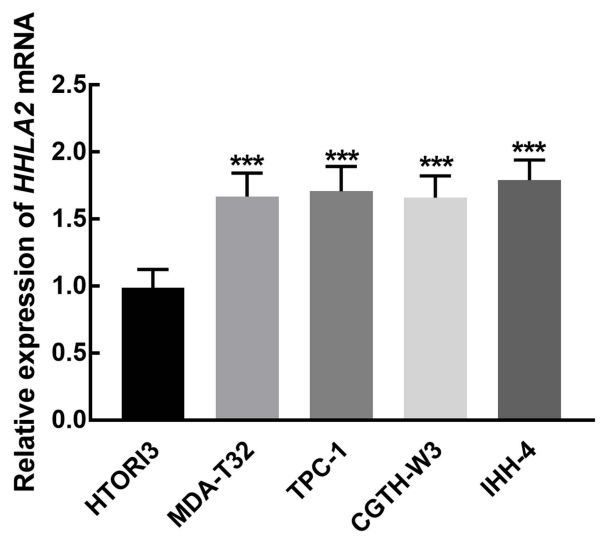

Figure I Expression of HHLA2 mRNA in PTC. (A) HHLA2 was significantly upregulated in PTC tissues. $* * * P<0.001$ relative to normal tissues. (B) The expression of HHLA2 in PTC cells (MDA-T32, TPC-I, CGTH-W3, and IHH-4 cell) was significantly high than that in normal cells (HTORI3 cell). ***P < $0.00 \mathrm{I}$.

\section{Statistical Analysis}

All data were expressed as mean value $\pm \mathrm{SD}$, obtained from triplicate experiments and analyzed with SPSS 20.0 software (IBM, USA). The difference between groups was estimated with Student's $t$-test one-way ANOVA followed by the Turkey post-hoc test. The clinical significance of HHLA2 was evaluated by the chi-square test, Kaplan-Meier, and Cox regression analysis. $P<0.05$ represents statistically significant difference.

\section{Results}

\section{HHLA2 Was Significantly Upregulated in PTC}

The expression level of HHLA2 mRNA was significantly higher in PTC tissues than that in normal tissues $(P<0.001$, Figure 1A). Consistently, the significant upregulation of HHLA2 was also found in PTC cells (MDA-T32, TPC-1, CGTH-W3, IHH-4 cells) in comparison with normal cells $(P<0.01$, Figure 1B). Moreover, the specific expression level of HHLA2 in TPC-1 and IHH-4 cells was relatively high than other cells, but the difference was not significant, indicating the relatively high sensitivity of these two cells to the upregulation of HHLA2. Therefore, the following in vitro cell experiments were conducted in these two cells.

\section{Clinical Significance of HHLA2 in PTC}

The PTC patients were partitioned into two groups with the average expression level of HHLA2 in PTC tissues as the cutoff, including a low-expression group consisting of 49 patients and a high-expression group consisting of 58 patients. Patients with high expression of HHLA2 always showed the positive status of lymph node metastasis and patients with advanced TNM stage were observed to possess a relatively high expression of HHLA2 (Table 1). The significant association between HHLA2 expression and lymph node metastasis $(P=0.020)$ and TNM stage $(P=0.006)$ was demonstrated, while no significant relationships were observed in other clinical features of included patients (Table 1).

Additionally, patients in the high-expression group were found to possess a relatively poorer survival rate than that of patients in the low-expression group, and the difference was significant ( $\log$ rank $P=0.020$, Figure 2). Furthermore, HHLA2 $(95 \% \mathrm{CI}=1.075-6.087, P=0.034)$ and the TNM stage $(95 \% \mathrm{CI}=1.012-6.093, P=0.047)$ were identified as two independent prognostic factors of patients with the HR value of 2.558 and 2.483, respectively (Table 2).

\section{The Biological Function of HHLA2 in PTC}

The expression of HHLA2 in PTC cells was regulated by the corresponding transfection. In TPC-1 (Figure 3A) and IHH4 cells (Figure 3B), HHLA2 expression was significantly suppressed by the transfection of HHLA2 shRNA and enhanced by the lentivirus containing HHLA2 sequences $(P<0.001)$. 
Table I Association Between HHLA2 Expression and Patients' Clinical Features Assessed by the Chi-Square Test

\begin{tabular}{|c|c|c|c|c|}
\hline & \multirow[t]{2}{*}{ Total $(n=107)$} & \multicolumn{2}{|c|}{ HHLA2 Expression } & \multirow[t]{2}{*}{$P$ value } \\
\hline & & Low $(n=49)$ & High $(n=58)$ & \\
\hline Age (years) & & & & 0.752 \\
\hline$\leq 45$ & 52 & 23 & 29 & \\
\hline$>45$ & 55 & 26 & 29 & \\
\hline Sex & & & & 0.811 \\
\hline Male & 45 & 20 & 25 & \\
\hline Female & 62 & 29 & 33 & \\
\hline LNM & & & & $0.020^{*}$ \\
\hline Negative & 59 & 33 & 26 & \\
\hline Positive & 48 & 16 & 32 & \\
\hline TNM stage & & & & $0.006 * *$ \\
\hline$|-| \mid$ & 68 & 38 & 30 & \\
\hline III-IV & 39 & II & 28 & \\
\hline Tumor size $(\mathrm{cm})$ & & & & 0.157 \\
\hline$\leq 4$ & 51 & 27 & 24 & \\
\hline$>4$ & 56 & 22 & 34 & \\
\hline Cancer foci & & & & 0.148 \\
\hline Single & 53 & 28 & 25 & \\
\hline Multiple & 54 & 21 & 33 & \\
\hline
\end{tabular}

Notes: $* P<0.05, * * P<0.01$ indicates the statistical significance.

Abbreviation: LNM, lymph node metastasis.

The proliferation of TPC-1 and IHH-4 cells was dramatically inhibited by the knockdown of HHLA2 and promoted by its overexpression $(P<0.05$, Figure 4A). Similarly, the promoted effect of HHLA2 upregulation was also observed in the migration (Figure 4B) and invasion (Figure 4C) of PTC cells, and the opposite effect was exerted by HHLA2 silencing $(P<0.001)$.

\section{Discussion}

Identification of biomarkers associated with tumor progression and clinical prognosis of patients is becoming a novel focus of cancer research. Previously, various molecules, such as non-coding RNAs and functional genes, have been characterized as indicators of cancer early detection and development prediction. ${ }^{13,14}$ HHLA2 is a novel identified member of the B7 family, a widely accepted immune checkpoint superfamily. ${ }^{15,16}$ The effects of HHLA2 on T-cell proliferation and cytokine production had been widely reported. ${ }^{10,17}$ However, the extra function of HHLA2 was elusive. Herein, the functional role and clinical significance of HHLA2 in PTC were estimated.

It was revealed that HHLA2 could co-express with PD-L1 in clear cell renal carcinoma and could predict the severity and prognosis of patients. ${ }^{18}$ In the present study, the significant upregulation of HHLA2 was observed in PTC. The upregulation of HHLA2 was notably associated with the lymph node metastasis status and TNM stage of patients, which are two important factors correlated with disease development, suggesting the involvement of HHLA2 in the progression 


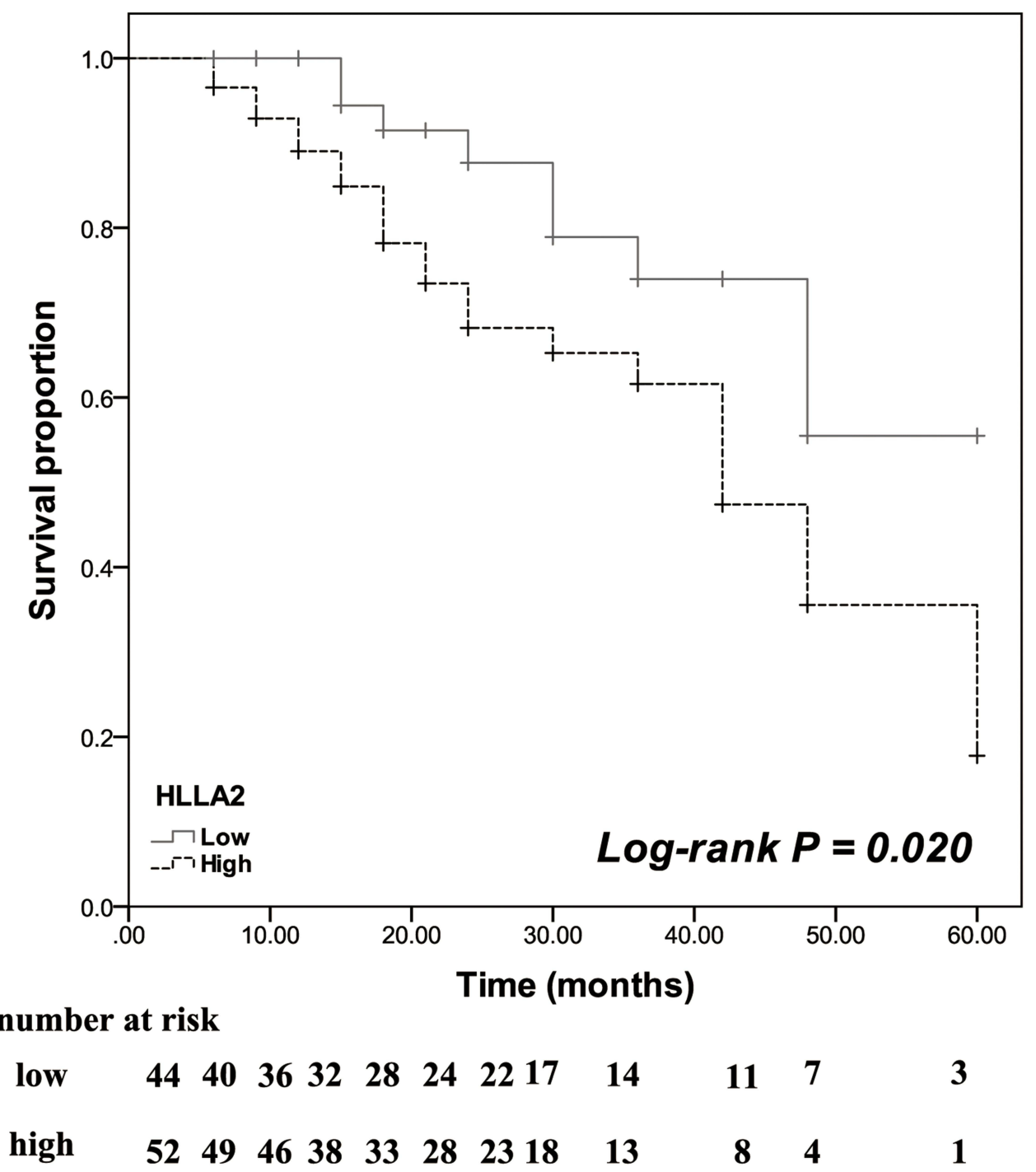

Figure 2 Kaplan-Meier curve of included patients. Patients with high expression of HHLA2 possessed a poor survival rate. Log rank $P=0.020$.

of PTC. Additionally, HHLA2 was also demonstrated to possess negative relationship with the survival rate of PTC patients. Previously, HHLA2 has also been identified as a prognostic indicator in various cancers. For instance, in epithelial ovarian cancer, HHLA2 behaves as a prognostic predictor associated with tumor differentiation and improved survival of patients. ${ }^{19}$ In hepatocellular carcinoma, the co-expression of HHLA2 and PD-L1 was infrequent, but the

Table 2 Association Between Clinical Features and Survival of Patients

\begin{tabular}{|l|l|l|l|}
\hline & HR Value & $\mathbf{9 5 \%} \mathbf{~ C l}$ & P value \\
\hline HHLA2 & 2.558 & $1.075-6.087$ & $0.034^{*}$ \\
Age & 1.405 & $0.684-2.885$ & 0.354 \\
Sex & 1.435 & $0.664-3.099$ & 0.358 \\
LNM & 1.586 & $0.738-3.405$ & 0.237 \\
TNM stage & 2.483 & $1.012-6.093$ & $0.047^{*}$ \\
Tumor size & 1.676 & $0.711-3.953$ & 0.238 \\
Cancer foci & 1.966 & $0.889-4.344$ & 0.095 \\
\hline
\end{tabular}

Note: $* P<0.05$ indicates the statistical significance.

Abbreviation: LNM, lymph node metastasis. 
A

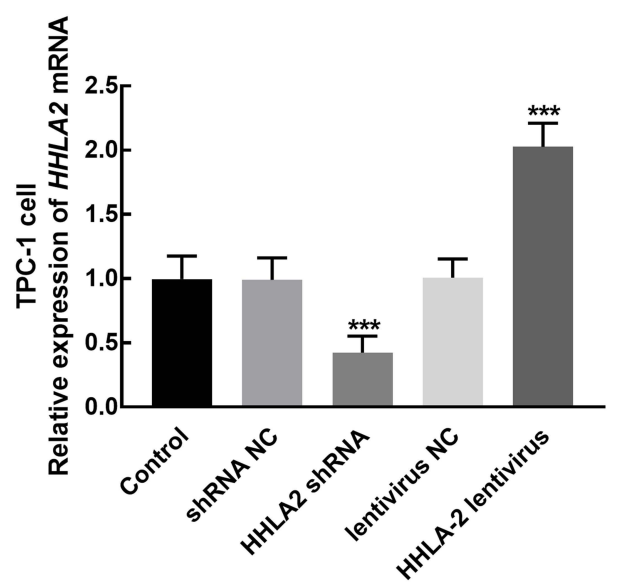

B

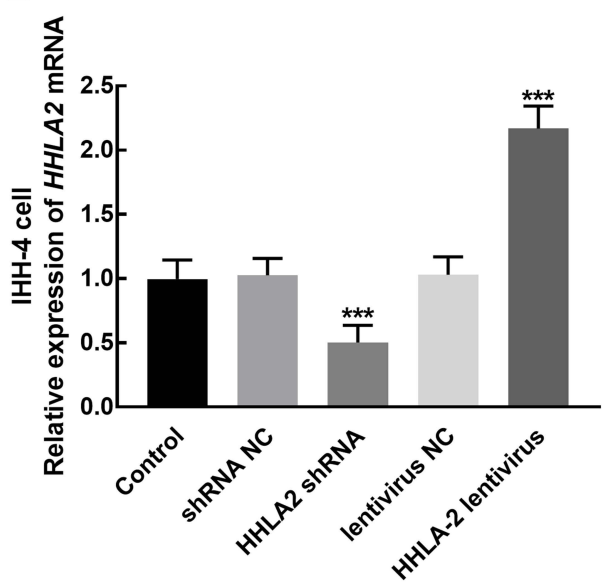

Figure 3 The expression of HHLA2 was regulated by cell transfection in TPC-I (A) and IHH-4 (B) cells. HHLA2 was significantly inhibited by the transfection of HHLA2 shRNA and enhanced by HHLA3 lentivirus. ${ }^{* * * P}<0.001$.

overexpression of HHLA2 showed significant prognostic value. ${ }^{20}$ However, the overall survival rate of enrolled patients in the present study was slightly different from previous data, ${ }^{21}$ which might be a result of the relatively small sample size. Therefore, a larger sample size is needed in future studies.

The biological effect of HHLA2 on the cellular processes of PTC was also assessed. The overexpression of HHLA2 was noticed to accelerate the progression of PTC cells, while the knockdown of HHLA2 exerted an opposite effect on the progression of PTC cells. These results suggested the tumor promoter role of HHLA2 in PTC. Early, the role of HHLA2 in the other cancers has also been disclosed, which is consistent with the obtained results. A similar upregulation of HHLA2 was observed in hepatocellular carcinoma, and the knockdown of HHLA2 increased cell adhesion, accelerated cell apoptosis, resulted in cell arrest and suppressed cellular processes. ${ }^{22}$ The overexpression of HHLA2 inhibited cell proliferation of ovarian cancer. ${ }^{19}$ The observed promoted effect of HHLA2 on PTC cells provides a view that the inhibition of HHLA2 might be a novel therapeutic strategy of PTC treatment.

However, this study did not leak out the mechanism underlying the function of HHLA2. In NSCLC, the EGFR/ MAPK/ERK signaling pathway was demonstrated to be the mechanism, through which HHLA2 regulated cell proliferation, migration, invasion, and cell cycle. ${ }^{23}$ The function of HHLA2 in immune processes has been widely reported, and numerous studies have focused on the role of immune regulation and immune escape in PTC. For example, a previous study identified a variety of dysregulated immune-related genes in PTC and established a risk model predicting the clinical outcome. ${ }^{24}$ Moreover, the role of HHLA2 in immune regulation also implies the potential of HHLA2 in immunotherapeutic approaches as well as in the therapy of PTC. ${ }^{15,25-27}$ Therefore, immune-related processes should attract special attention, which might be a potential mechanism that mediates the tumor promoter role of HHLA2 in PTC. ${ }^{10,26}$ Further mechanism investigations that would leak out the signaling and receptors of HHLA2 are warranted, which would be a part of the future studies.

\section{Conclusion}

Taken together, the upregulation of HHLA2 in PTC is associated with the advanced development and poor prognosis of patients. HHLA2 functions as a tumor promoter that enhances the proliferation, migration, and invasion of PTC cells. These findings provide a novel insight into the clinical management of PTC. 

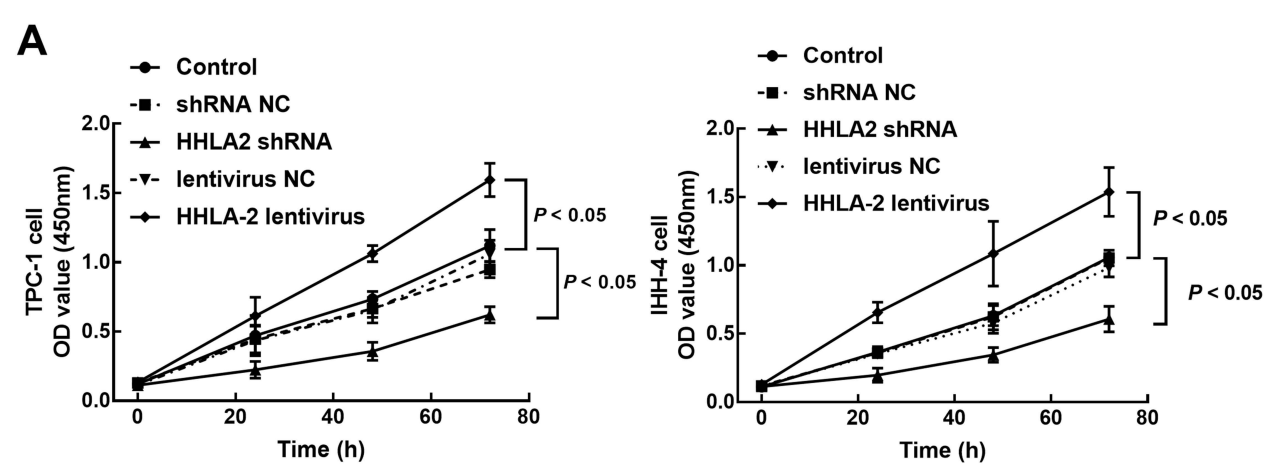

B
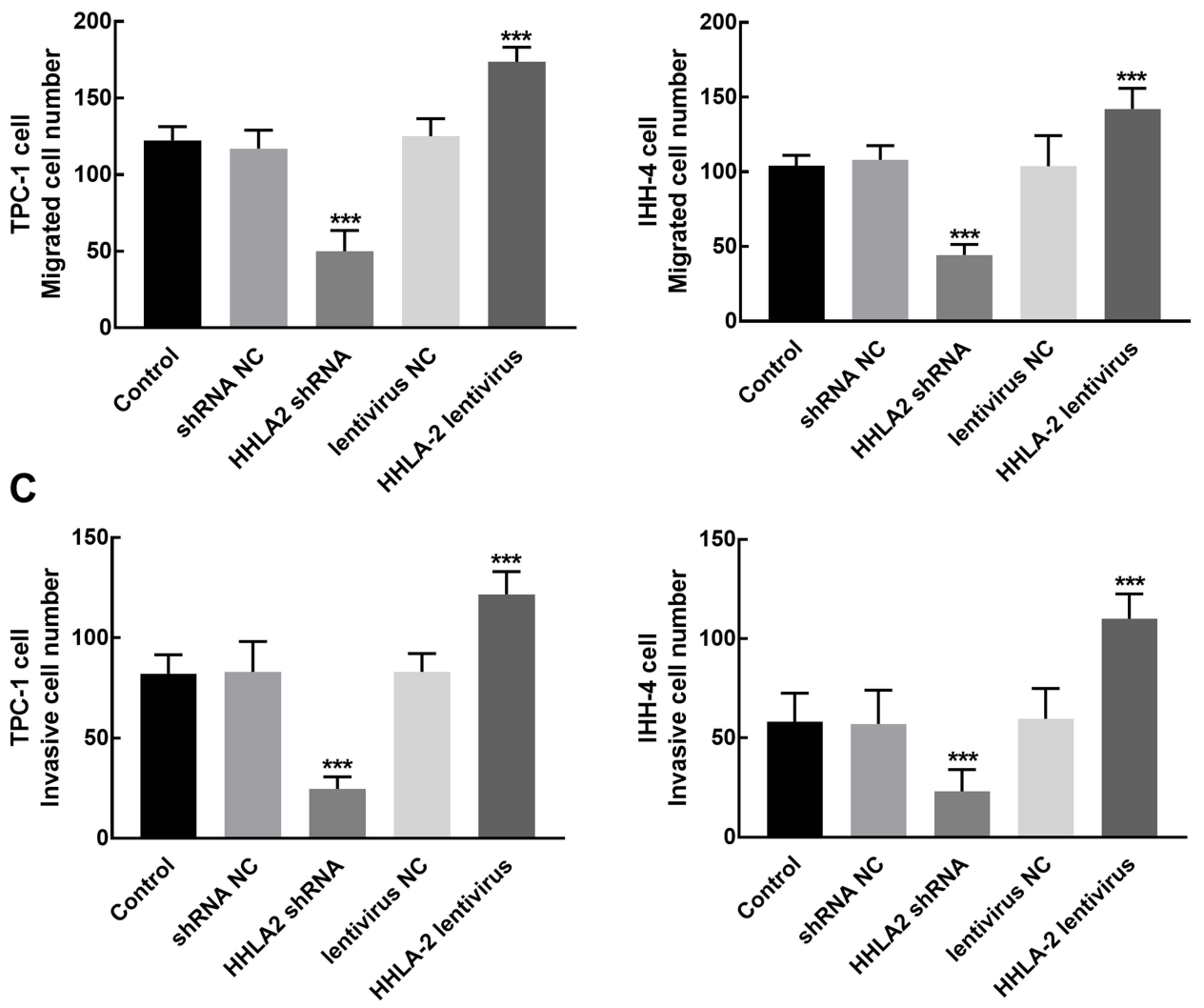

Figure 4 Effect of HHLA2 on the proliferation, migration, and invasion of PTC cells. (A) The overexpression of HHLA2 significantly promoted the proliferation of TPC-I and IHH-4 cells, which was suppressed by its knockdown. (B) The migration of TPC-I and IHH-4 cells was enhanced by HHLA2 upregulation and inhibited by HHLA2 silencing. (C) The invasion of TPC-I and IHH-4 cells was enhanced by HHLA2 upregulation and inhibited by HHLA2 silencing. ${ }^{* * * P}<0.00 \mathrm{I}$.

\section{Disclosure}

The authors report no conflicts of interest in this work.

\section{References}

1. Cabanillas ME, McFadden DG, Durante C. Thyroid cancer. Lancet. 2016;388(10061):2783-2795. doi:10.1016/S0140-6736(16)30172-6

2. Abdullah MI, Junit SM, Ng KL, Jayapalan JJ, Karikalan B, Hashim OH. Papillary thyroid cancer: genetic alterations and molecular biomarker investigations. Int J Med Sci. 2019;16(3):450-460. doi:10.7150/ijms.29935

3. Asa SL. The current histologic classification of thyroid cancer. Endocrinol Metab Clin North Am. 2019;48(1):1-22. doi:10.1016/j.ecl.2018.10.001

4. Carling T, Udelsman R. Thyroid cancer. Annu Rev Med. 2014;65:125-137. doi:10.1146/annurev-med-061512-105739

5. LiVolsi VA. Papillary thyroid carcinoma: an update. Mod Pathol. 2011;24(Suppl 2):S1-S9. doi:10.1038/modpathol.2010.129

6. Grogan RH, Kaplan SP, Cao H, et al. A study of recurrence and death from papillary thyroid cancer with 27 years of median follow-up. Surgery. 2013;154(6):1436-46; discussion 1446-7. doi:10.1016/j.surg.2013.07.008 
7. Kakudo K, Tang W, Ito Y, Mori I, Nakamura Y, Miyauchi A. Papillary carcinoma of the thyroid in Japan: subclassification of common type and identification of low risk group. J Clin Pathol. 2004;57(10):1041-1046. doi:10.1136/jcp.2004.017889

8. Liang L, Jiang Y, Chen JS, et al. B7-H4 expression in ovarian serous carcinoma: a study of 306 cases. Hum Pathol. 2016;57:1-6. doi:10.1016/j. humpath.2016.06.011

9. Dong Z, Zhang L, Xu W, Zhang G. EGFR may participate in immune evasion through regulation of B7-H5 expression in non-small cell lung carcinoma. Mol Med Rep. 2018;18(4):3769-3779. doi:10.3892/mmr.2018.9361

10. Zhao R, Chinai JM, Buhl S, et al. HHLA2 is a member of the B7 family and inhibits human CD4 and CD8 T-cell function. Proc Natl Acad Sci US A. 2013;110(24):9879-9884. doi:10.1073/pnas.1303524110

11. Wei L, Tang L, Chang H, Huo S, Li Y. HHLA2 overexpression is a novel biomarker of malignant status and poor prognosis in gastric cancer. Hum Cell. 2020;33(1):116-122. doi:10.1007/s13577-019-00280-2

12. Boor PPC, Sideras K, Biermann K, et al. HHLA2 is expressed in pancreatic and ampullary cancers and increased expression is associated with better post-surgical prognosis. Br J Cancer. 2020;122(8):1211-1218. doi:10.1038/s41416-020-0755-4

13. Romano G, Veneziano D, Acunzo M, Croce CM. Small non-coding RNA and cancer. Carcinogenesis. 2017;38(5):485-491. doi:10.1093/carcin/ bgx026

14. Hristova VA, Chan DW. Cancer biomarker discovery and translation: proteomics and beyond. Expert Rev Proteomics. 2019;16(2):93-103. doi:10.1080/14789450.2019.1559062

15. Janakiram M, Shah UA, Liu W, Zhao A, Schoenberg MP, Zang X. The third group of the B7-CD28 immune checkpoint family: HHLA2, TMIGD2, B7x, and B7-H3. Immunol Rev. 2017;276(1):26-39. doi:10.1111/imr.12521

16. Flem-Karlsen K, Fodstad O, Nunes-Xavier CE. B7-H3 immune checkpoint protein in human cancer. Curr Med Chem. 2020;27(24):4062-4086. doi:10.2174/0929867326666190517115515

17. Zhu Y, Yao S, Iliopoulou BP, et al. B7-H5 costimulates human T cells via CD28H. Nat Commun. 2013;4(1):2043. doi:10.1038/ncomms3043

18. Zhou QH, Li KW, Chen X, et al. HHLA2 and PD-L1 co-expression predicts poor prognosis in patients with clear cell renal cell carcinoma. J Immunother Cancer. 2020;8(1):e000157. doi:10.1136/jitc-2019-000157

19. Xu G, Shi Y, Ling X, et al. HHLA2 predicts better survival and exhibits inhibited proliferation in epithelial ovarian cancer. Cancer Cell Int. 2021;21 (1):252. doi:10.1186/s12935-021-01930-y

20. Jing CY, Fu YP, Yi Y, et al. HHLA2 in intrahepatic cholangiocarcinoma: an immune checkpoint with prognostic significance and wider expression compared with PD-L1. J Immunother Cancer. 2019;7(1):77. doi:10.1186/s40425-019-0554-8

21. Milano AF. Thyroid cancer: 20-year comparative mortality and survival analysis of six thyroid cancer histologic subtypes by age, sex, race, stage, cohort entry time-period and disease duration (SEER*Stat 8.3.2) a systematic review of 145,457 cases for diagnosis years $1993-2013$. J Insur Med. 2018;47(3):143-158. doi:10.17849/insm-47-03-143-158.1

22. Luo M, Xiong Y, Lin Y, Liang R, Li Y, Ge L. H Long Terminal Repeat-Associating 2 (HHLA2) is a biomarker of advanced stage hepatocellular carcinoma and promotes tumor cell development in vitro. Med Sci Monit. 2021;27:e930215. doi:10.12659/MSM.930215

23. Sun W, Li S, Tang G, et al. HHLA2 deficiency inhibits non-small cell lung cancer progression and THP-1 macrophage M2 polarization. Cancer Med. 2021;10(15):5256-5269. doi:10.1002/cam4.4081

24. Gan X, Guo M, Chen Z, et al. Development and validation of a three-immune-related gene signature prognostic risk model in papillary thyroid carcinoma. J Endocrinol Invest. 2021;44(10):2153-2163. doi:10.1007/s40618-021-01514-7

25. Yan H, Qiu W, Koehne de Gonzalez AK, et al. HHLA2 is a novel immune checkpoint protein in pancreatic ductal adenocarcinoma and predicts post-surgical survival. Cancer Lett. 2019;442:333-340. doi:10.1016/j.canlet.2018.11.007

26. Wei Y, Ren X, Galbo PM Jr, et al. KIR3DL3-HHLA2 is a human immunosuppressive pathway and a therapeutic target. Sci Immunol. 2021;6(61). doi:10.1126/sciimmunol.abf9792

27. Castellanos JR, Purvis IJ, Labak CM, et al. B7-H3 role in the immune landscape of cancer. Am J Clin Exp Immunol. 2017 ;6(4):66-75.

International Journal of General Medicine

Dovepress

\section{Publish your work in this journal}

The International Journal of General Medicine is an international, peer-reviewed open-access journal that focuses on general and internal medicine, pathogenesis, epidemiology, diagnosis, monitoring and treatment protocols. The journal is characterized by the rapid reporting of reviews, original research and clinical studies across all disease areas. The manuscript management system is completely online and includes a very quick and fair peer-review system, which is all easy to use. Visit http://www.dovepress.com/testimonials.php to read real quotes from published authors.

Submit your manuscript here: https://www.dovepress.com/international-journal-of-general-medicine-journal 